\title{
Equation of state and neutrino signal from collapsing stellar cores
}

\section{A. Yudin* and D. Nadyozhin}

A.I. Alikhanov Institute for Theoretical and Experimental Physics

E-mail: yudin@itep.ru, nadezhineitep.ru

During the infall stage of collapse up to nuclear densities, the properties of stellar matter are well described by the Nuclear Statistical Equilibrium (NSE) approach . Even with this simplification there still exists a variety of effects in the Equation Of State (EOS) to be taken into account. We start from the problem of nuclear partition functions then continue with a nuclear network completeness question, closely connected with a neutron-rich nuclei problem. Separate studies are devoted to the problem of Coulomb interaction in multi-component systems. Our final goal is to explore these effects and to estimate their impact on the structure of core-collapse supernovae and the properties of the neutrino signal they produced.

International Symposium on Nuclear Astrophysics - Nuclei in the Cosmos - IX

25-30 June 2006

CERN

${ }^{*}$ Speaker. 


\section{Introduction}

Under the condition of NSE, the properties of EOS are determined by only three parameters: density $\rho$, temperature $T$, and neutron-to-proton ratio $\theta$. In addition to these explicit parameters, there exist a number of "degrees of freedom" which are connected to the microphysics involved. For example, one has to specify the set of nuclei (nuclear network) to be explicitly included in the calculations. Thus, there arises the problem of nuclear network completeness. One must be sure that the nuclear network specified accurately reproduce both chemical and thermodynamic properties of matter. After some numerical experiments we settled on the network that includes free neutrons and protons, the set of $\alpha$-nuclides from ${ }^{4} \mathrm{He}$ through ${ }^{40} \mathrm{Ca}$, and isotopes of iron-peak elements from Sc through $\mathrm{Kr}$ (in total 137 nuclides). If necessary, we supplement this basic network with a group of the neutron-rich nuclei.

\section{Nuclear partition functions}

The correct evaluation of nuclear partition functions is a long-standing problem. Our approach [1] is similar to that of Rauscher et al. [2] except the high-temperature domain. At high temperatures, our approach satisfies to a natural requirement that the average excitation energy of a nucleus $\left\langle E_{\mathrm{ex}}\right\rangle$ should not exceed it's binding energy $Q_{\mathrm{A}, \mathrm{Z}}$. Hence, for the high-temperature domain we use the relation $\left\langle E_{\mathrm{ex}}\right\rangle=Q_{\mathrm{A}, \mathrm{Z}} \chi(T)$. In principle, one can use an arbitrary form for the monotonously increasing with temperature function $\chi(T)$ that satisfies the condition $0 \leq \chi(T) \leq 1$. Then, by integrating the relation $\left\langle E_{\mathrm{ex}}\right\rangle=T \frac{d \ln \omega}{d \ln T}$ one can obtain the partition function $\omega$. The results are shown in Fig. 1. The left panel shows the partition function of ${ }^{56} \mathrm{Fe}$ as a function of temperature. Red line represents our approach, blue line corresponds to the calculations of Rauscher et al., and black one shows the well-known Bethe's formula. Right panel shows the corresponding average excitation energies. One can see that for the approaches of Bethe and Rauscher et al. $\left\langle E_{\mathrm{ex}}\right\rangle$ exceeds the binding energy already at $T_{9} \gtrsim 100$ indicating that the corresponding partition functions are inapplicable for such a high temperature. Our method allows to estimate the partition functions in a reasonable way even at very high temperatures. This is especially important for the case of the neutron-rich nuclei when the binding energy is often the only parameter known.

And what is the effect of the nuclear excitations on the thermodynamic properties of matter? One result is shown in Fig. 2 where the level lines of of the adiabatic index are plotted. One can see that calculation with an allowance for the nuclear excitations (solid lines) results in a widening of upper part of the instability ravine - the domain where adiabatic index $\gamma<4 / 3$ (the ravine edge is shown by a thick red lines).

\section{Neutron-rich nuclei}

The importance of the neutron-rich nuclei for the advanced stages of the collapse have been confirmed by many authors. These nuclei are significant not only for elucidating the chemical composition and thermodynamical properties described by EOS but also for a correct description of the kinetics of nuclear weak-interactions that control the temporal behavior of $\theta$ during the collapse. Figure 3 shows how such important thermodynamic quantities as dimensionless entropy 

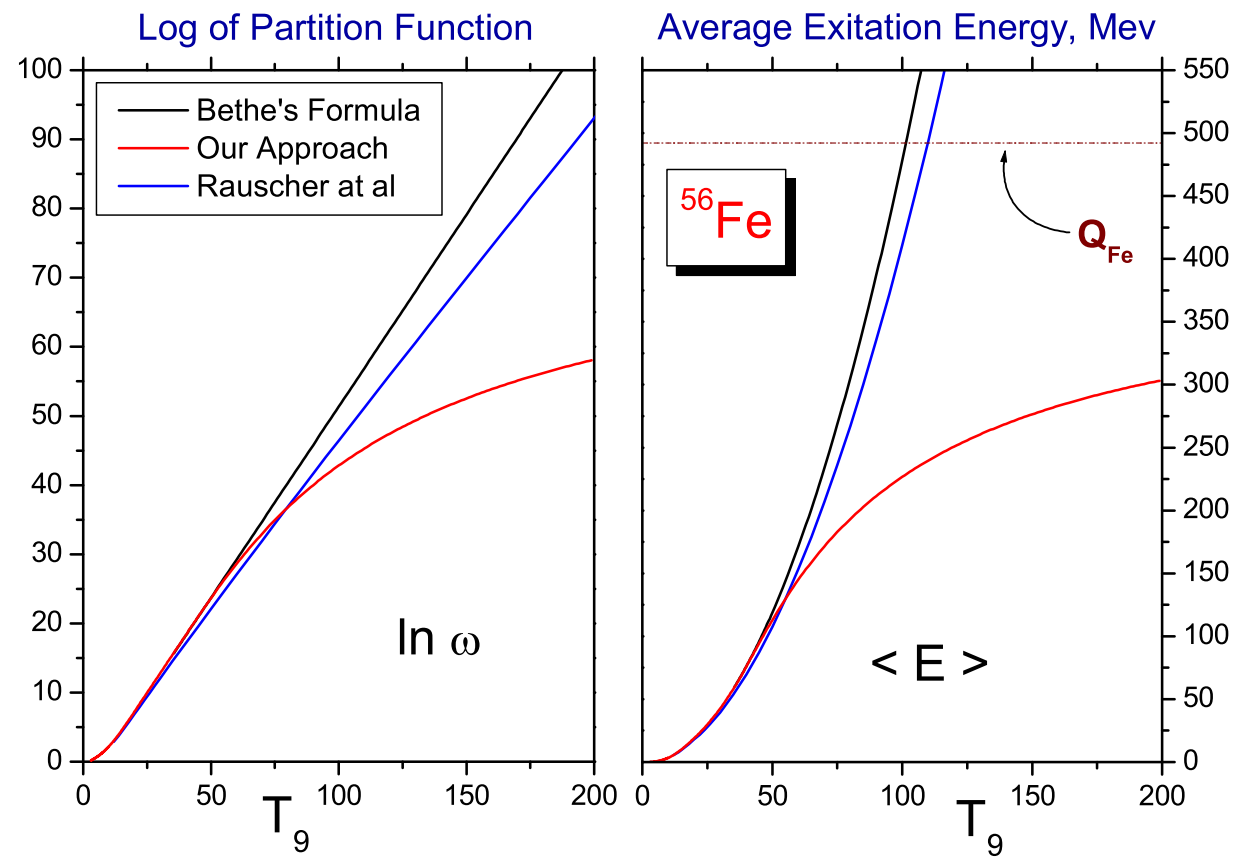

Figure 1: Average excitation energy and partition function for ${ }^{56} \mathrm{Fe}$.

per nucleon and adiabatic index vary with $\theta$. Dashed lines represents the calculation carried out with the standard set of nuclei mentioned above. Solid red lines correspond to the calculation with seven neutron-rich isotopes of $\mathrm{Se}\left({ }^{83-89} \mathrm{Se}\right)$ being added to the standard set. Until $\boldsymbol{\theta}$ does not exceed its individual value 1.44 for ${ }^{83} \mathrm{Se}$ the addition of ${ }^{83-89} \mathrm{Se}$ virtually makes no difference. However, for $\theta>1.5$ the difference becomes significant and can even destabilize those regions which were stable against the collapse $(\gamma>4 / 3)$.

\section{Coulomb Interaction}

To account for the Coulomb interaction under the conditions of NSE is a rather complicated problem because it influences not only the thermodynamic quantities but also can significantly change the condition of chemical equilibrium and thereby the nuclide abundances $Y_{i}$. The free energy formalism is the most adequate for this issue. The free energy of the system with interaction is represented as a sum of free energy without interaction (ideal part) and extra term, determined by the Coulomb interaction: $F\left(\rho, T,\left\{Y_{i}\right\}\right)=F_{\text {id }}\left(\rho, T,\left\{Y_{i}\right\}\right)+\triangle F\left(\rho, T,\left\{Y_{i}\right\}\right)$. The new set of equilibrium concentrations can be found by minimization of $F\left(\rho, T,\left\{Y_{i}\right\}\right)$ with respect to $Y_{i}$ at constant $T, \rho$, and $\theta$.

First of all, one must specify a form of the interaction-free part $\triangle F$ and then address to the problem of Coulomb interaction in a multi-component system. The most known and carefully studied is the case of one-component system where the interaction term has the form $\triangle F_{\text {OCS }}=$ $n k T f(\Gamma)$, where $f$ is a universal function of the only one parameter $\Gamma=\frac{(Z e)^{2}}{k T}\left(\frac{4 \pi}{3} n\right)^{1 / 3}, n$ being 


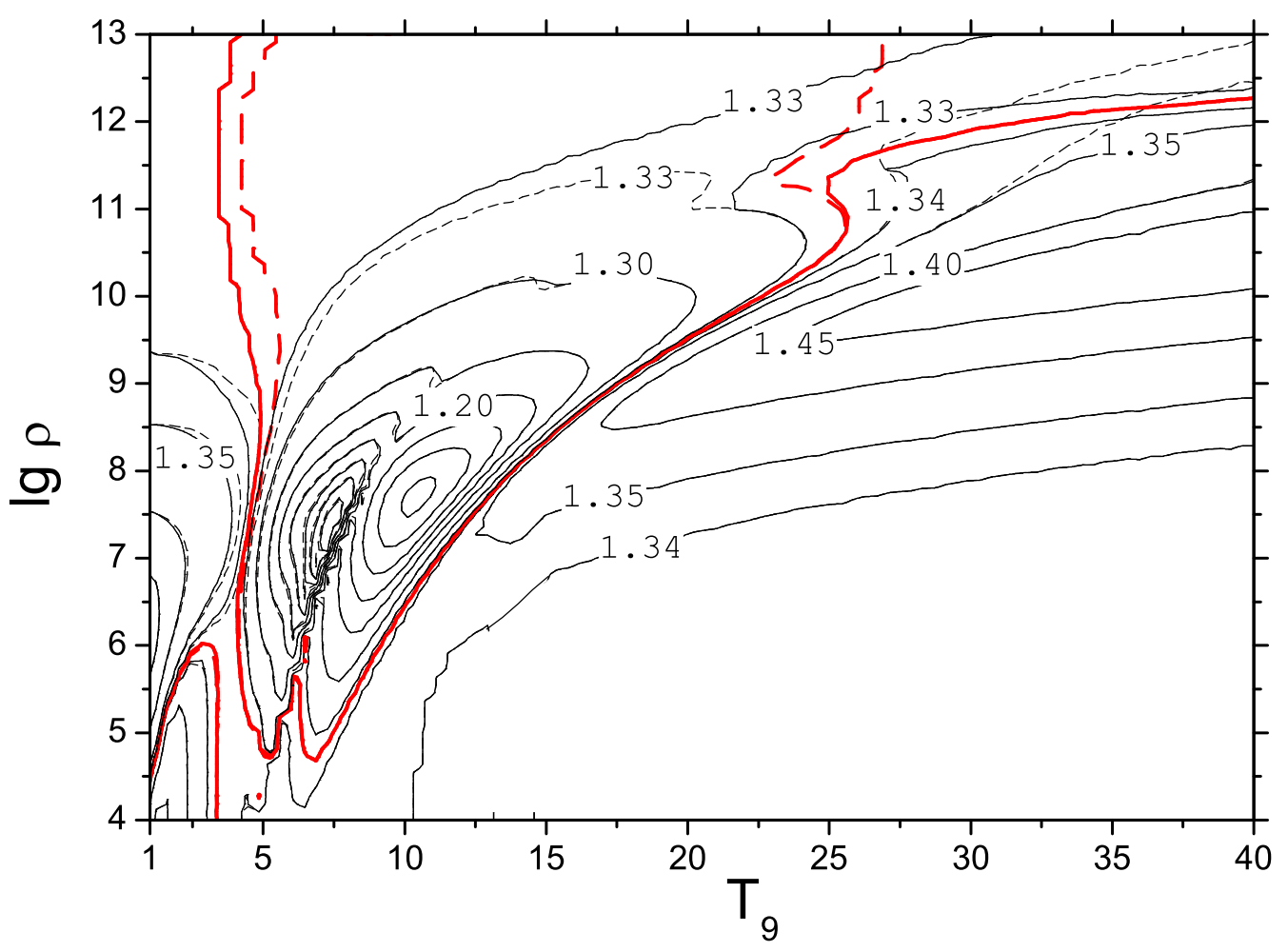

Figure 2: Level lines of adiabatic index $\gamma$ with (solid lines) and without (dashed lines) nuclear excitation.

the number of particles per unit volume and $Z e$ their individual charge. There exist two wellknown limits: the limit of weak interaction (the Debye limit) and that of strong interaction called linear mixing rule. In the latter case the interaction part of free energy can be represented as $\triangle F_{\mathrm{LMR}}=k T \sum n_{i} f\left(\Gamma_{i}\right), \Gamma_{i}=Z_{i}^{5 / 3} \Gamma_{e}$, where $\Gamma_{e}$ is the electron interaction factor. Thus, the linear mixing rule is defined as a linear sum over the component concentrations and uses the same onecomponent function $f(\Gamma)$ for all the component involved. The validity of this rule in the domain of strong interaction have been confirmed by many authors. But for astrophysical implementation we need an approach that would be applicable in the whole domain of the interaction.

The simplest way to reach such an aim is to use the average-ion model. It based on the onecomponent model with all the quantities involved being averaged over chemical components, i.e. $\triangle F_{\mathrm{AIM}}=k T\left(\sum n_{i}\right) f(\langle\Gamma\rangle),\langle\Gamma\rangle=\sum n_{i} \Gamma_{i} / \sum n_{i}$. Using this approach one can cover both strong and weak-interaction limits. However, such an approach always fails to adequately describe intermediate interaction regime $\langle\Gamma\rangle \sim 1$ causing nonphysical behavior of equilibrium concentrations and thermodynamic quantities (there appear oscillations and even breaks). Thus we came to the conclusion that various forms of average-ion approach is inapplicable to describe the Coulomb-interaction effects under the conditions of NSE.

The other way is to start from the linear-mixing rule and to try to expand its applicability to weak interaction. But doing so in the spirit of average-ion model, i.e. modifying it by some additional parameters averaged over chemical components, one comes to the same difficulties. After some logical attempts and numerical experiments we have found a special modification technique 

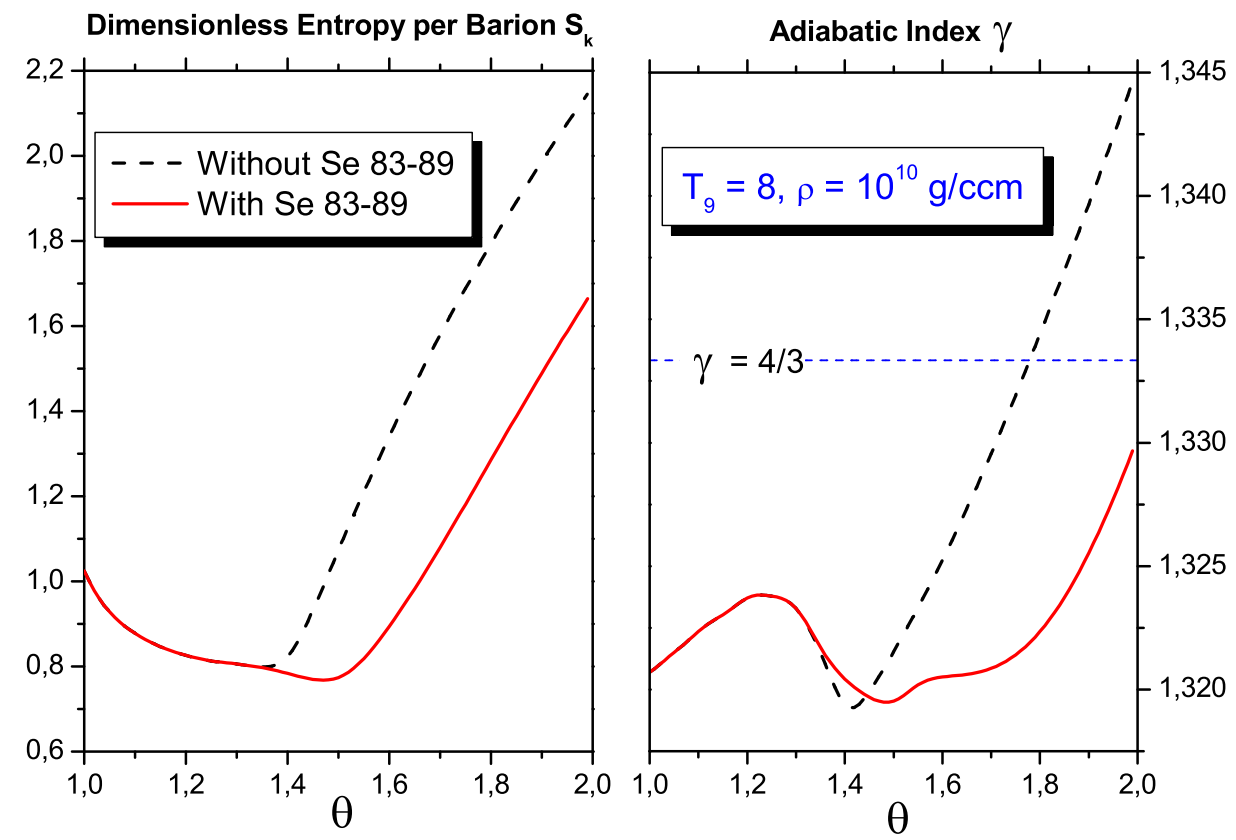

Figure 3: Influence of neutron-rich nuclei on the entropy (left panel) and adiabatic index (right panel).

which is free from the problems listed above and predicts smooth behavior for all the essential quantities [3].

Why is this intermediate interaction region so important? Figure 4 gives the answer. It shows the moment after the bounce when the shock wave just has begun to propagate outwards from stellar center. We have calculated the average interaction factor $\langle\Gamma\rangle$ for three cases: EOS without nuclear excitation (black dashed line), with excitation (red solid line) and with Coulomb interaction and excitation (blue solid line). The central region and that above the shock wave belong to strong interaction domain $\Gamma>1$, the region passed by the shock belongs to the weak interaction domain. The transition between them occurs somewhere at the shock front and one needs the correct description of this important region.

\section{Neutrino signal}

The spectra and light curves of neutrino radiation are the most important issues of any collapse calculation. Here we present our first results of the calculations for a $2 M_{\odot}$ stellar core collapse. These calculations were done without an allowance for Coulomb interaction. Figure 5 represents the calculated neutrino light curves for the electron neutrino $v_{\mathrm{e}}$. The first luminosity maximum occurs at the point 2 approximately $1.25 \mathrm{~ms}$ before the bounce (point 1 ) and is due to the temperature increase during the infall. The second peak $\sim 1.38 \mathrm{~ms}$ after bounce is caused by a rapid expansion of shock front emitting surface. The average emitted neutrino energy that was smoothly growing before the bounce begins rapidly to rise after it up to $15 \mathrm{MeV}$. This effect is caused by changes 


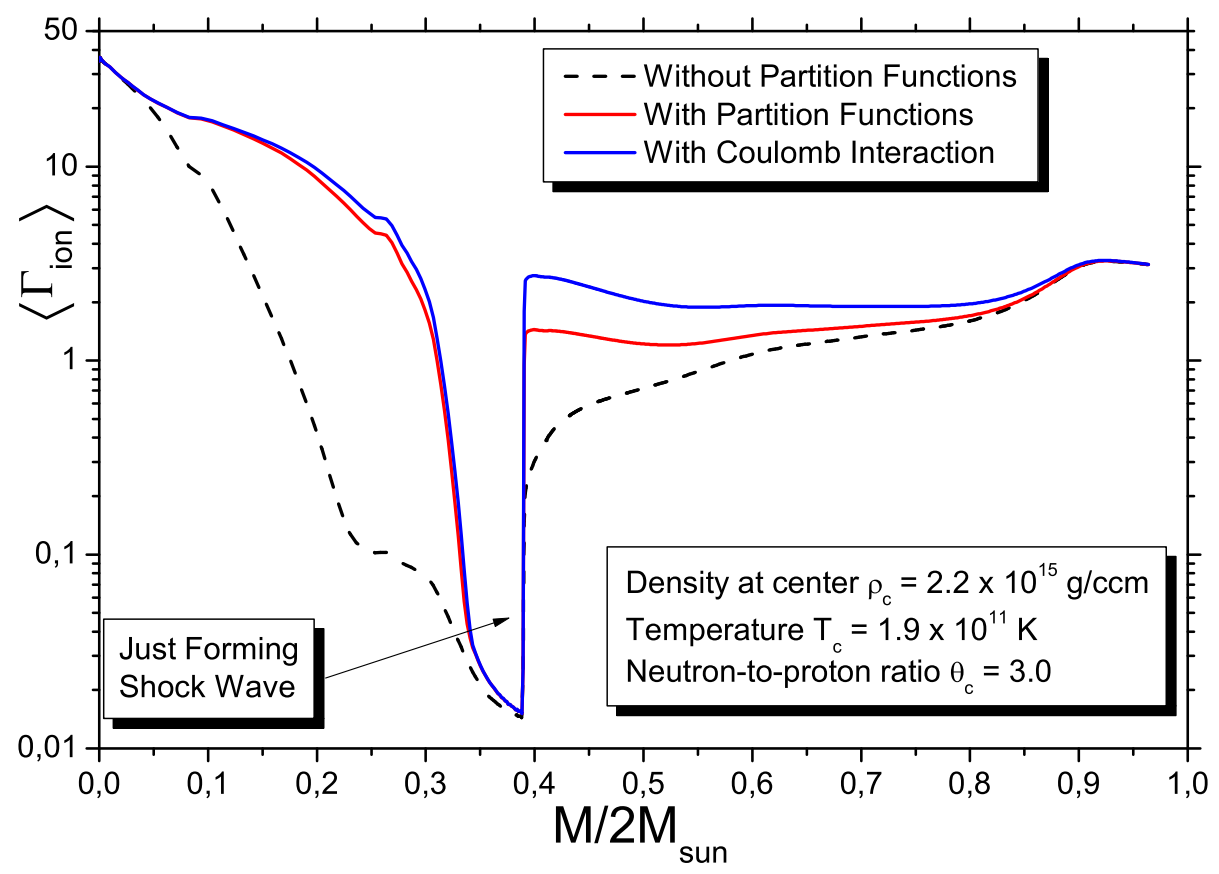

Figure 4: Coulomb interaction factor as a function of mass coordinate.

in the structure of collapsing core in the vicinity of moving shock wave: behind the shock there appears a layer filled with free neutrons and protons. After that both the neutrino luminosity and the mean individual $v_{\mathrm{e}}$ energy begin to decrease. As for the electron antineutrino $\widetilde{v}_{\mathrm{e}}$, these values continue to increase smoothly. However, the contribution of $\widetilde{v}_{\mathrm{e}}$ to the total neutrino flux is small. The total, emitted in form $v_{\mathrm{e}}$ and $\widetilde{v}_{\mathrm{e}}$ energy at $15 \mathrm{~ms}$ after bounce reaches $\approx 10^{52} \mathrm{erg}$.

Figure 6 shows the spectra of $v_{\mathrm{e}}$ (red solid line) and $\widetilde{v}_{\mathrm{e}}$ (magenta line) at the moment of bounce $(0 \mathrm{~ms})$ and $5 \mathrm{~ms}$ later. The calculation neglecting the effect of the neutrino-electron scattering (NES) are shown by a black dashed line.

At the moment of bounce, NES noticeably shifts spectrum peak to lower energies as compared to the non-NES calculations. At $5 \mathrm{~ms}$ after bounce this effect is less pronounced, the spectrum gets stiffer and broader.

The final goal of our study is to explore how the properties of the neutrino signal depend on different physical effects especially such as the Coulomb interaction, partition functions, and properties of the neutron-rich nuclei. This work is underway.

Acknowledgement. We thank the NIC-9 Organizing Committee for the invitation to attend the Conference. The work was supported by the grants: RFBR No. 04-02-16793 and SNSF No. IB7320-110996. 


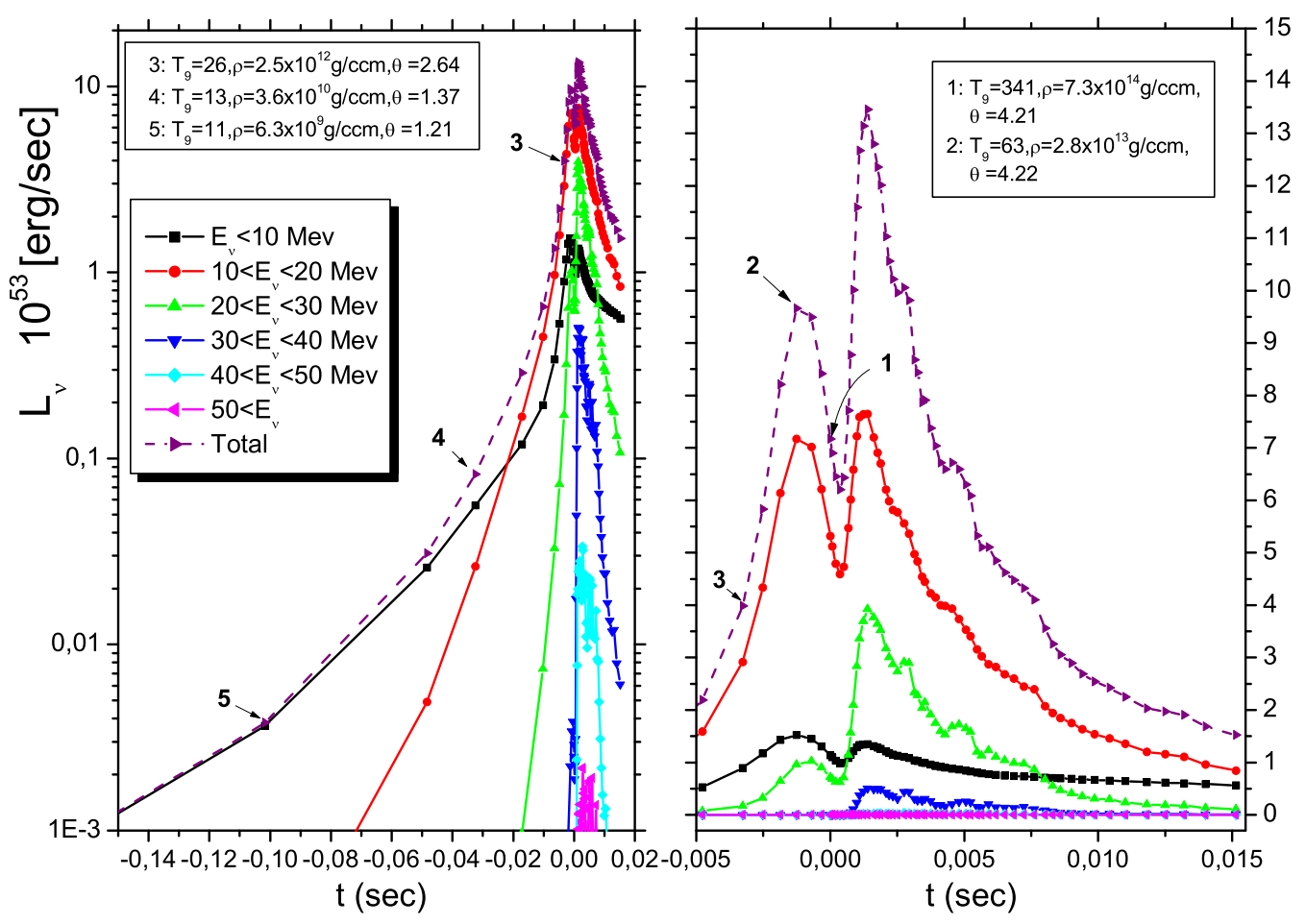

Figure 5: The electron neutrino light curves in different energy groups. The total (energy-integrated) neutrino luminosity is shown by purple solid triangles, (left panel, upper curve). The right panel shows the near-peak region $-0.005<t<0.015$ in more detail $(t=0$ corresponds to the moment of bounce). 
A. Yudin

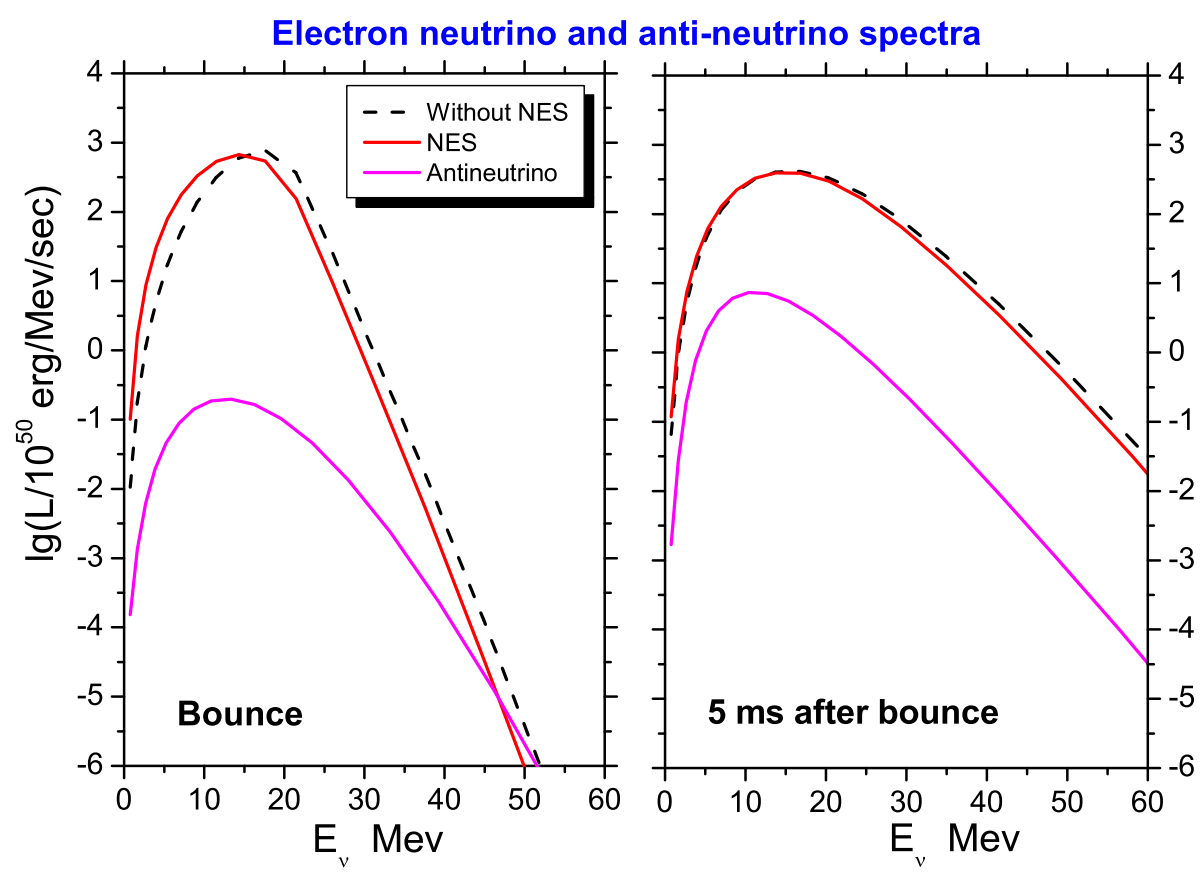

Figure 6: Electron neutrino and antineutrino spectra.

\section{References}

[1] D.K. Nadyozhin and A.V. Yudin, Equation of State under Nuclear Statistical Equilibrium Conditions, Pis'ma Astron. Zh. 30, 697 (2004) [Astron. Lett. 30, 634 (2004)].

[2] T. Rauscher, F.K. Thielemann, and K.L. Kratz, Nuclear Level Density and the Determination of Thermonuclear Rates for Astrophysics, Phys.Rev. C 56, 163 (1997).

[3] D.K. Nadyozhin and A.V. Yudin, The Influence of Coulomb Interaction on the Equation of State under Nuclear Statistical Equilibrium Conditions, Pis'ma Astron. Zh., 31, 4 (2005) [Astron. Lett. 31, 4 (2005)]. 